\title{
Liver abscess caused by fish bone perforation of Meckel's diverticulum: a case report
}

Seiji Natsuki, Yasuhito Iseki ${ }^{*}$ Hishashi Nagahara, Tatsunari Fukuoka, Masatsune Shibutani and Masaichi Ohira

\begin{abstract}
Background: Liver abscess due to gastrointestinal perforation by foreign bodies is rare. Furthermore, there are few case reports of liver abscess via the portal vein caused by perforation of the lower gastrointestinal tract by a foreign body.

Case presentation: A 54-year-old man visited our hospital because of a fever that had lasted for 1 month. There were no physical findings except for the fever. Laboratory tests showed only elevated inflammatory markers. Abdominal contrast-enhanced computed tomography revealed an abscess in the right lobe of the liver and a highdensity object in the small intestine. We diagnosed him with liver abscess secondary to intestinal perforation by a foreign body. The patient underwent drainage of the liver abscess and laparoscopic surgery for perforation of the small intestine. A fish bone had perforated the top of Meckel's diverticulum, which had been covered by the ileal mesentery. We successfully performed diverticulectomy and removed the fish bone. The patient was discharged without complications on the 13th postoperative day.
\end{abstract}

Conclusions: Liver abscess caused by foreign bodies requires multidisciplinary treatment, so we must detect and remove the cause of the abscess earlier. Liver abscess can form via the portal vein secondary to lower gastrointestinal perforation, as in this case. When exploring the cause of liver abscess, we should investigate the whole body, including the lower gastrointestinal tract.

Keywords: Liver abscess, Fish bone, Foreign body perforation, Meckel's diverticulum

\section{Background}

Foreign bodies in the gastrointestinal (GI) tract are often encountered in clinical practice. Most ingested foreign bodies pass through uneventfully, and the incidence of intestinal perforation after foreign body ingestion is approximately $1 \%$ [1]. Foreign body perforation usually occurs due to fishbones, chicken bones, toothpicks, needles, or pens [2]. In Japan, foreign body perforation is often caused by fish bones [3]. Reported etiologies of fish bone perforation include (1) ingestion of a fish bone

\footnotetext{
* Correspondence: m2074067@med.osaka-cu.ac.jp

Department of Gastroenterological surgery, Osaka City University Graduate School of Medicine, 1-5-7 asahimachi, abeno-ku, osaka-shi, Osaka-fu 545-8586, Japan
}

longer than the luminal diameter, (2) dysmotility due to abdominal adhesions, and (3) diverticula or hernia [4].

The most common etiologies of liver abscess include (1) complications of cholangitis, (2) bloodstream dissemination via the portal vein and hepatic artery in systemic sepsis, (3) local spread from infected contiguous tissue, and (4) traumatic injury [5, 6]. Liver abscess secondary to foreign body perforation is extremely rare $[7,8]$. There have been several case reports of liver abscess due to foreign body in the GI tract penetrating the liver directly. There are also a few case reports of liver abscess via the portal vein caused by perforation of the lower GI tract by a foreign body. 
We herein report a case of liver abscess secondary to Meckel's diverticulum perforation induced by a fish bone.

\section{Case presentation}

A 54-year-old man visited his primary doctor with a fever. He was prescribed common cold medicine, but his fever persisted. After visiting several hospitals, he was referred to the department of general practice at our hospital due to a 1-month history of a spiking fever. $\mathrm{He}$ had a medical history of hypertension. On admission, his body temperature was $37.5^{\circ} \mathrm{C}$. The findings from a physical examination were unremarkable, and there was no abdominal pain, nausea, or abdominal distension. The white blood cell count was $17,600 / \mu \mathrm{L}(80.5 \%$ neutrophils), and the C-reactive protein level was $7.66 \mathrm{mg} / \mathrm{dL}$. Serum bilirubin, aspartate aminotransferase, and alanine aminotransferase levels were within normal range. Chest and abdominal X-ray findings were unremarkable; however, abdominal contrast-enhanced computed tomography $(\mathrm{CT})$ revealed an abscess in the right lobe of the liver (segment 8) and a high-density linear object in the small intestine with fat stranding. There were no free air and other abdominal abscess (Fig. 1). On admission, blood cultures grew Streptococcus oralis.

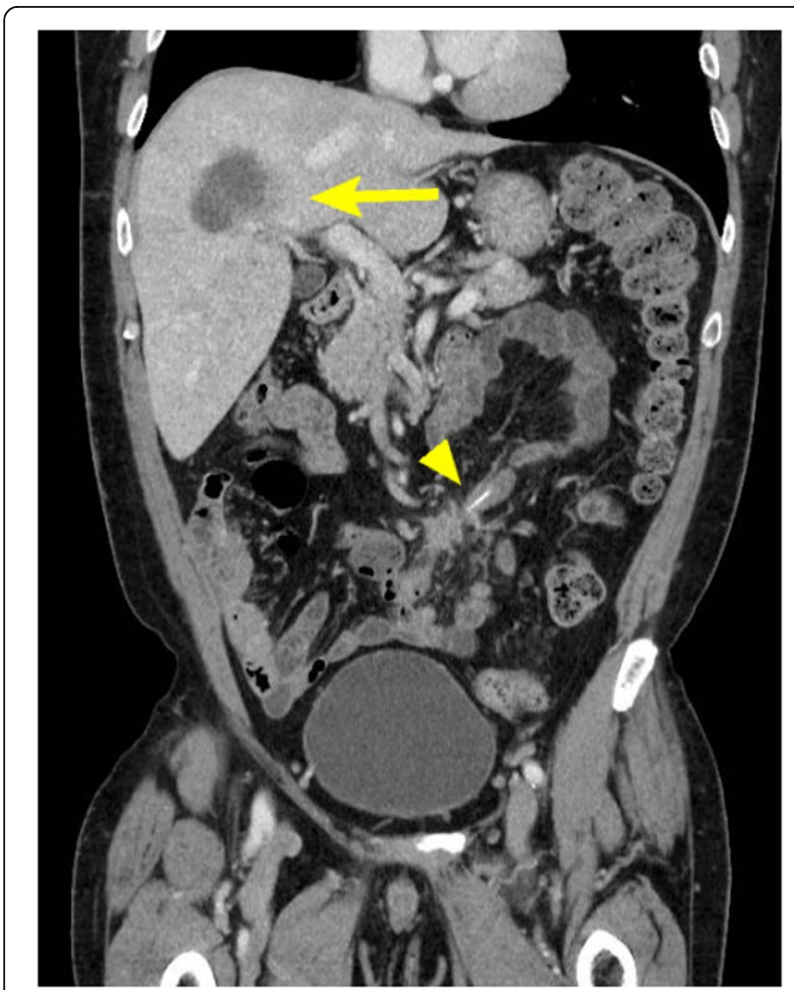

Fig. 1 Enhanced computed tomography $(C T)$ revealed an abscess in the right lobe of the liver and a needle-like foreign body in the small intestine with fat stranding
The general practitioner suspected this to be a case of fish bone bowel perforation with liver abscess, and a surgical consult was thus requested. The patient often ate fish but did not recall whether or not he had swallowed any fish bones. The liver abscess was about $5 \mathrm{~cm}$ in size, so we drained it by a percutaneous approach and administered ceftriaxone and metronidazole. Purulent fluid cultures from the liver abscess grew Streptococcus intermedius. He had no abdominal symptoms, so he underwent double-balloon endoscopy (DBE) before surgery. We observed the lower ileum with DBE and the intestine downstream from the ileal end by X-ray fluoroscopy, but we were unable to detect any foreign bodies, diverticula, or leakage.

We subsequently performed laparoscopic surgery due to suspicion of fish bone perforation. Laparoscopy showed that there was no ascites or abdominal abscess. Meckel's diverticulum was located in the ileum approximately $50 \mathrm{~cm}$ upstream from the ileal end and had adhered to the ileal mesentery with inflammation. On peeling away the adhesion, we found a $2-\mathrm{cm}$ fish bone perforating the top of Meckel's diverticulum that had been covered by mesentery. There were no other abnormal findings of the intestine (Fig. 2). We thus performed diverticulectomy. In addition, we did not need to drain the liver abscess laparoscopically.

The histopathological report showed Meckel's diverticulum without ectopic tissues. Recovery after the surgery was uneventful, and he was discharged on the 13th postoperative day.

\section{Discussion and conclusions}

Most ingested foreign bodies are excreted without injuring the GI tract, and only $1 \%$ of people who ingest foreign bodies experience any symptoms, such as perforation [1]. The most common sites of perforation by foreign bodies are, in descending order, the ileum, transverse colon, and sigmoid colon [9]. The present patient had Meckel's diverticulum presenting with fish bone perforation. Meckel's diverticulum is the most common congenital malformation of the GI tract, occurring in $2-4 \%$ of the population [10]. Most patients with Meckel's diverticulum are asymptomatic, but a few may develop GI bleeding, abdominal pain, bowel obstruction, or perforation. Proposed causes of Meckel's diverticulum perforation include ectopic mucosa, a foreign body, inflammatory bowel disease (IBD), and tumors [11]. There have been several reports of Meckel's diverticulum perforation due to a fish bone; however, almost all cases developed peritonitis and underwent surgery within a few days. In our case, the Meckel's diverticulum with perforation induced by a fish bone had become covered by the mesentery, leading to localized inflammation. We therefore believe that our patient developed liver abscess 


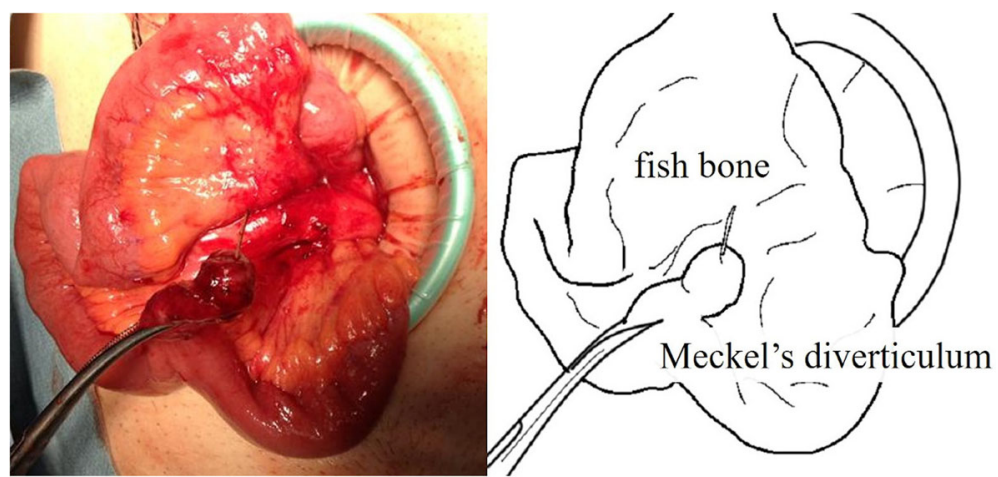

Fig. 2 There was a fish bone perforating the top of the Meckel's diverticulum, which had been covered by mesentery

secondarily after the symptom had persisted for 1 month.

Our patient had no abdominal symptoms, and we suspected that the ingested foreign body might have been able to be removed by endoscopy. For that reason, we performed DBE. However, we were unable to detect any lesions via DBE, including foreign bodies, diverticula, and malignancy. On an examination of the resected specimen, the Meckel's diverticulum showed a 1-cm orifice; however, this orifice might have been difficult to detect using DBE.

At surgery, we detected a fish bone perforating the top of the Meckel's diverticulum, but the species of fish was

Table 1 Reports on liver abscess caused by fish bone perforation

\begin{tabular}{|c|c|c|c|c|c|c|c|}
\hline Case & Year of publication & Author & Age (years) & Sex & Abscess portion & Perforation portion & How to form liver abscess \\
\hline 1 & 1990 & Aoki & 73 & Male & Right lobe & unknown & unknown \\
\hline 2 & 1993 & Tamura & 61 & Male & Left lobe & unknown & unknown \\
\hline 3 & 1995 & Matsuzaki & 56 & Male & Right lobe & duodenum & directly \\
\hline 4 & 1995 & Kato & 73 & Female & Left lobe & stomach & directly \\
\hline 5 & 1995 & Mimoto & 50 & Male & Left lobe & stomach & directly \\
\hline 6 & 1999 & Horii & 61 & Male & Left lobe & unknown & directly \\
\hline 7 & 2005 & Oda & 38 & Male & Left lobe & stomach & directly \\
\hline 8 & 2006 & Roki & 77 & Male & Left lobe & stomach & directly \\
\hline 9 & 2006 & Mizunuma [3] & 53 & Female & Right lobe & duodenum & directly \\
\hline 10 & 2007 & Nagai & 54 & Male & Left lobe & stomach & directly \\
\hline 11 & 2007 & Kadowaki & 73 & Male & Left lobe & duodenum & directly \\
\hline 12 & 2008 & Clarençon & 64 & Male & Right lobe & unknown & directly \\
\hline 13 & 2009 & Matsuo & 74 & Female & Left lobe & stomach & directly \\
\hline 14 & 2010 & Kataoka & 64 & Female & Left lobe & unknown & unknown \\
\hline 15 & 2011 & Ohara & 81 & Male & Left lobe & stomach & directly \\
\hline 16 & 2013 & Akimori & 72 & Male & Left lobe & stomach & directly \\
\hline 17 & 2014 & Mukaihashi & 68 & Male & Left lobe & stomach & directly \\
\hline 18 & 2015 & Ishikawa & 63 & Male & Right lobe & unknown & unknown \\
\hline 19 & 2015 & Hosoi & 83 & Female & Left lobe & stomach & directly \\
\hline 20 & 2016 & Nagashima [13] & 62 & Male & Right lobe & ileum & via portal vein \\
\hline 21 & 2017 & Urata & 75 & Female & Left lobe & duodenum & directly \\
\hline 22 & 2018 & Kohama & 74 & Male & Left lobe & unknown & directly \\
\hline \multirow[t]{2}{*}{23} & 2019 & Tsutsumi & 41 & Male & Left lobe & stomach & directly \\
\hline & & Our case & 54 & Male & Right lobe & ileum & via portal vein \\
\hline
\end{tabular}

To our knowledge, there has been only one case report with a similar presentation [13]; that report described a case of liver abscess secondary to fish bone perforation via the portal vein 
not identified. Most previous reports on cases of Meckel's diverticulum perforation due to a fish bone also failed to identify the species of fish [12]. The present patient often ate fish of different species and was thus unable to recall which species might have led to his condition.

Klebsiella pneumoniae is the most common pathogen causing liver abscess. However, in our case, blood cultures grew S. oralis, and purulent fluid cultures from the liver abscess grew $S$. intermedius. These are the same genus and constitute part of the normal bacterial flora of the human mouth, nasopharynx, and GI tract. Both cultures grew the same bacterial genus, which was not Klebsiella, and the patient developed fish bone perforation; we therefore diagnosed the liver abscess as secondary to fish bone perforation via the portal vein. Malignant neoplasm in the lower GI has been reported to cause liver abscess via the portal vein because of (1) malignancy inhibiting immunity, (2) destruction of the intestinal wall barrier, or (3) increasing pressure in the colon causing the intestinal flora to be transported into vessels [13]. However, there have been quite a few reports fish bone perforation in the lower GI tract causing liver abscess. As shown in Table 1, several case reports have described liver abscess due to a foreign body in the GI tract directly penetrating the liver; to our knowledge, however, there is only one case report with a similar presentation to ours [13]. In that case, the patient had recurrent episodes of liver abscess and a fish bone penetrating the ileum in the right lower abdomen. Blood cultures grew S. oralis, and drainage cultures from the liver abscess isolated $S$. intermedius, just as in our case.

Liver abscess is a life-threatening infection, and source control is important for the management of abdominal sepsis [14]. All abscesses more than $4 \mathrm{~cm}$ in size should be managed by percutaneous drainage and antibiotics $[5$, 6]. Treating liver abscess due to non- $K$. pneumoniae with empirical antibiotics targeting to $K$. pneumoniae has also been suggested [5]. Therefore, in our case, we performed percutaneous drainage and administered ceftriaxone and metronidazole.

Liver abscess cases due to foreign bodies require multidisciplinary treatment, so we must detect and remove the cause of the liver abscess as early as possible [15]. In addition, when exploring the cause of liver abscess, we should investigate the whole body, including the lower GI tract.

\section{Abbreviations}

$\mathrm{CT}$ : Computed tomography; DBE: Double balloon endoscopy;

Gl: Gastrointestinal; IBD: Inflammatory bowel disease

\section{Acknowledgements}

The authors would like to thank Japan Medical Communication (https:// www.japan-mc.co.jp/) for the English language review.
Authors' contributions

SN acquired the data and drafted the manuscript. YI, TF, MS, HN and MO were involved in drafting in the manuscript. MO was the chief supervisor. All authors have read and approved the final manuscript.

\section{Funding}

This research didn't receive any grant from funding agencies in the public, commercial, or not-for-profit sectors.

Availability of data and materials

Not applicable.

Ethics approval and consent to participate

Not applicable.

Consent for publication

The authors obtained the written informed consent for publication from this patient.

\section{Competing interests}

The authors declare that they have no competing interests.

Received: 18 December 2019 Accepted: 27 May 2020

Published online: 05 June 2020

References

1. Henderson FF, Gaston EA. Ingested foreign body in the gastrointestinal tract. Arch Surg. 1938;36:66-95.

2. McCanse DE, Kurchin A, Hinshaw JR. Gastrointestinal foreign bodies. Am J Surg. 1981;142:335-7.

3. Mizunuma K, Nakamura H, Fujitaka T, et al. A case of hepatic abscess that formed secondary to fish bone penetration. Jpn J Gastroenterol Surg. 2006; 39:1811-5.

4. Oikawa M, Ishikawa $H$, Akama $H$, et al. Fish bone perforations of the gastrointestinal tract. J Abdom Emerg Med. 2007;27(3):441-6.

5. Shelat VG, Wang Q, Chia CL, et al. Patients with culture negative pyogenic liver abscess have th e same outcomes compared to those with Klebsiella pneumoniae pyogenic liver abscess. Hepatobiliary Pancreat Dis Int. 2016; 15(5):504-11.

6. Ahmed S, Chia CLK, Junnarkar SP, et al. Percutaneous drainage for giant pyogenic liver abscess—is it safe and sufficient? Am J Surg. 2016;211(1):95-101.

7. $\mathrm{Ng} \mathrm{CT}$, Htoo A, Tan SY. Fish bone-induced hepatic abscess: medical treatment. Singap Med J. 2011;52:e56-8.

8. Santos SA, Alberto SC, Cruz E, et al. Hepatic abscess induced by foreign body: case report and literature review. World J Gastroenterol. 2007;13: 1466-70.

9. Yoh K, Inoue $S$, Watanebe $Y$, et al. Intestinal perforation by fish bone: case report and review of 271 cases in the Japanese literature. Jpn J Gastroenterol Surg. 2001;34:1640-4.

10. Turgeon DK, Barnett JL. Meckel's diverticulum. Am J Gastroenterol. 1990;85: 777-81.

11. Shinohara T, Hirose $K$, Saji $Y$, et al. A case of perforation of meckel's diverticulum by a fish bone. J J.jpn Surg Assoc. 2004;65(9):2405-8.

12. Ishikawa $S$, Inayoshi A, Motomura $Y$, et al. A fish bone which penetrated the small intestine from a Meckel diverticulum. Jpn J Gastroentero Surg. 2012; 45(2):210-7

13. Nagashima K, Shou T, Haneda M, et al. A case report of a recurrent abscess secondary to fish bone penetration of the ileum. J Gastroenterol. 2016;113: 451-6.

14. Sartelli M, Chichom-Mefire A, Labricciosa FM, et al. The management of intra-abdominal infections from a global perspective: 2017 WSES guidelines for management of intra-abdominal infections. World J Emerg Surg. 2017; 12:29.

15. Bekki T, Fujikuni N, Amano H, et al. Liver abscess caused by fish bone perforation of stomach wall treated by laparoscopic surgery: a case report. Surg Case Rep. 2019;79(5):1-5.

\section{Publisher's Note}

Springer Nature remains neutral with regard to jurisdictional claims in published maps and institutional affiliations. 